\title{
OPTIMIZING INSPECTION STRATEGIES FOR MULTI-STAGE MANUFACTURING PROCESSES USING SIMULATION OPTIMIZATION
}

\author{
Vahid Sarhangian \\ Abolfazl Vaghefi \\ Dept. of Industrial Engineering \\ Iran University of Science \& Technology \\ Tehran, 16844, IRAN
}

Hamidreza Eskandari

School of Industrial Engineering

Tarbiat Modares University

Tehran, IRAN

\author{
Mostafa K. Ardakani
}

\author{
Dept. of Civil Engineering \\ The Catholic University of America \\ Washington, D.C. 20064, U.S.A.
}

\begin{abstract}
This paper deals with the problem of determining the optimal inspection strategy for a multi-stage production process using simulation optimization. An optimal inspection strategy is the one that results in the lowest total inspection cost, while still assuring a required output quality. Because of the complexity of the problem, simulation is used to model the multi-stage process subject to inspection and to calculate the resulting inspection costs. Simulation optimization is then used to find the optimal inspection strategy. The performance of the proposed method is presented through the use of a numerical example.
\end{abstract}

\section{INTRODUCTION}

Manufacturing systems are generally consisted of several stages, in which, raw materials pass through various operations and eventually transform into finished products.

Each processing stage will produce a proportion of items that fail to meet the necessary requirements. A preliminary idea to maintain the quality level is providing an inspection station after the last stage. This is generally referred to as outgoing inspection. However, with outgoing inspection, all efforts and costs invested in producing defective items during previous stages are wasted. It is more reasonable to place inspection stations after every major manufacturing process to insure that a specific quality level is being maintained.

Efficient economic inspection strategies minimize the total inspection cost while ensuring the required output quality. In other words, a tight inspection results in higher product quality, but will also lead to higher costs of inspection, scrap and rework. An optimum inspection plan balances these effects.

After each manufacturing stage, a full or sampling inspection can be performed. In full $(100 \%)$ inspection, all of the items in a lot are inspected and defective items are replaced or reworked. However, in sampling inspection, a sample is picked and inspected from the lot and based on the defective items observed in the sample, the lot can be rejected or accepted. Accepted lots are released to the next stage and rejected lots are submitted for full inspection. Good units replace all of the defectives found during the sampling or $100 \%$ inspection. Thus, in a multi-stage manufacturing system, the inspection strategy addresses the number and location of inspection stations and inspection parameters (sample size, acceptance number) for each inspection station.

Most multi-stage inspection models have focused on $100 \%$ inspection or screening, where defects occur independently and costs are proportional to the number of defects detected at each stage. Lindsay and Bishop (1964) showed that if the cost functions for inspection and reworking are assumed linear and the fraction of defective units for each stage is assumed to be fixed, then the $100 \%$ inspection will be more efficient than sampling. The similar results were found by White (1969) in the case where the rejected items are repaired and replaced with good items. Raz (1986) reviewed the previous researches on multi-stage inspection allocation and found out that when the inspection costs are concave, then the optimal level at each stage is probably either $100 \%$ or $0 \%$. On the other hand, Montgomery (2005) presented several situations in which sampling is most likely to be useful. For example, when the cost of $100 \%$ inspection is high and/or inspections typically need destructive testing, sampling is preferred. Heredia-Langner et al. (2002) presented a highly constrained multi-stage inspection problem where all stages must receive partial rectifying inspection and they solved their problem by using a real-valued Genetic Algorithm. Volsem et. al (2007) used simulation to model the multi-stage inspection problem and found the optimal inspection strategy by an Evolutionary Algorithm. Their method is able to determine which type of inspection $(0 \%$, $100 \%$ or sampling) should be performed in each stage and the rigor of the inspections. However, in their research sampling parameters are considered fixed.

Wu et al. (2001) pointed out in real cases; many unavoidable factors (such as the wear of tools and the fluc- 
tuation of power supply) may result in non-homogeneous lots with different defection rates. Therefore, it can be more appropriate to consider the proportion of defective items of each lot as a random variable. Wu et al. (2001) presented two optimum rectifying inspection plans where incoming fraction defective is a beta random variable. However, their optimal inspection plans were designed for a single-stage rather than a multi-stage process. It is easy to show that the optimum inspection plan for each stage does not necessarily guarantee the global optimum inspection plan for the multi-stage manufacturing system. A more comprehensive study can be carried out to use their method in multi-stage manufacturing systems.

This paper presents a modeling and solution approach based on simulation modeling and optimization to deal with the problem of determining the optimum inspection strategy for a given $n$-stage manufacturing system. For each stage, defective ratio is assumed to be a beta random variable. In this study, Arena simulation software is utilized to build the simulation model. We then use OptQuest, Arena built-in optimization package, to find the optimum solution. The model determines the optimum inspection strategy so that the expected total inspection cost including test cost, rework cost and penalty cost, is minimal.

The rest of the paper is organized as follows: In Section 2, we present the problem statement, mathematical model and the required theoretical background. The simulation model and the solution approach are presented in Section 3. We discuss the results through a numerical example in Section 4. Finally, conclusions are provided in Section 5.

\section{PROBLEM FORMULATION}

Consider a multi-stage manufacturing system, in which products pass through $r$ existing stages and inspection of products is performed immediately after each process stage. As shown in Figure 1, at each stage, a manufacturing process is carried out on the products before moving to the next possible inspection station. At each inspection station, $n$ items are randomly sampled from a lot of size $N$ $(n \leq N)$ and the number of defective item $d$ is determined. If $\bar{d}$ is less than or equal to a predetermined number, $c,(d$ $\leq c)$ defective items in the sample are replaced with good items and the lot would be accepted and released to the next manufacturing stage. However, if $d>c$, the entire lot is screened and all defective items are either reworked or replaced with acceptable ones. Such inspections are often called rectifying inspections (Montgomery 2005).

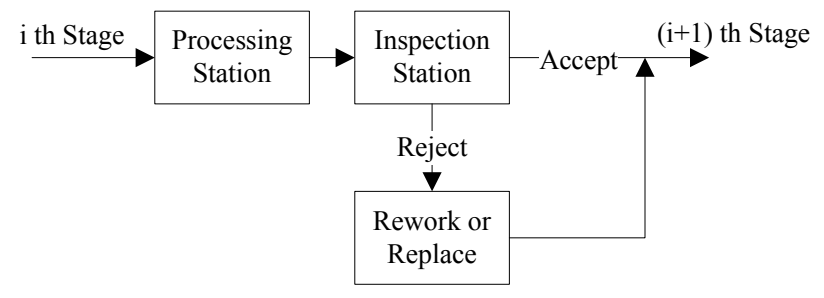

Figure 1: A manufacturing stage

\subsection{Model Assumptions}

In order to analyze the problem, the following assumptions are considered. The proportion of defective items inherently produced at stage $i$ denoted by $P^{0}{ }_{i}$ does not depend on earlier stages and can be adequately represented by a transformed beta distribution. Therefore, probability density function of $P^{0}{ }_{i}$ is:

$$
f\left(p_{i}^{0} \mid a, b\right)=\frac{\Gamma(a+b)}{\Gamma(a) \Gamma(b)}\left(p_{i}^{0}\right)^{a-1}\left(1-p_{i}^{0}\right)^{b-1},
$$

where $\Gamma$ (.) is gamma function and $0 \leq P^{0}{ }_{i} \leq 1$. As shown in Figure 2, a wide range of $P^{0}{ }_{i}$ can be conveniently represented by varying shape parameters $a$ and $b$ of the beta distribution. $P^{0}{ }_{i}$ can be rescaled and relocated to obtain a beta random variable on $\left[P_{w}, P_{l}\right]$ of the same shape by the transformation $P_{l}+\left(P_{u}-P_{l}\right) P_{i}^{0}$. Considering uncertainty in the proportion of defective items provides a more realistic model particularly, in the case that $P^{0}{ }_{i}$ is not exactly known and precise. Moreover, the exact sampling distribution of $d$ is the hyper-geometric distribution. Although for large lot size, binomial distribution can be employed as a good approximation for the hyper-geometric, we use the exact sampling distribution. This distribution produces more accurate results than does the binomial distribution.

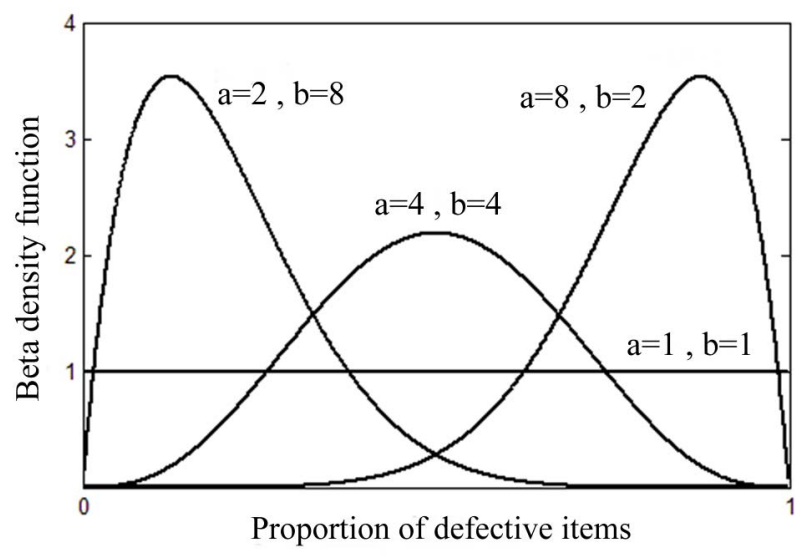

Figure 2: Beta distribution with different parameters 


\subsection{Notations and Mathematical Formulation}

Prior to further model development, the following notations are adopted.

- $\quad N_{i}$ : lot size at stage $i$.

- $P_{i}^{0}$ : proportion of defective items inherent to stage $i$.

- $\quad P_{i}$ : actual proportion of defective items of stage $i$.

- $n d_{i}$ : average number of defective items, detected at stage $i$.

- $\quad t c_{i}$ : test cost per item at stage $i$.

- $\quad p c$ : penalty cost per defective item after stage $n$.

- $\quad r c_{i}$ : rework cost per item at stage $i$.

- TTC: total test cost.

- TRC: total rework cost.

- TPC: total penalty cost.

- TIC: total inspection cost.

It is assumed that all rejected items are reworked and replaced with good items. Therefore, $\mathrm{Ni}^{\text {'s }}$ are identical and equal to $N$ for all stages. In this model, the sample size and the acceptance number are the decision variables and can be defined as follows:

- $n_{i}$ : sample size, the number of items to be inspected at stage $i$,

- $\quad c_{i}$ : maximum number of defective items that can be accepted in a given sample at stage $i$.

For the first stage of a multi-stage process, the actual proportion of defective items $P_{i}$ is equal to $P_{i}^{0}$. For the following stages $i(i=2, \ldots, r)$, however, $P_{i}$, also depends on the inspection strategy chosen on the previous stage(s) and can be calculated as follows:

$$
P_{i}=P_{i}^{0}+\mathrm{AOQ}_{i-1}-\left(P_{i}^{0} \times \mathrm{AOQ}_{i-1}\right),
$$

where the average outgoing quality for stage $i\left(\mathrm{AOQ}_{i}\right)$ refers to average outgoing proportion of defectives out of stage $i$ over the accepted and rejected lots, with $\mathrm{AOQ}_{0}=0$. Notice that it is assumed that $P_{i}$ and $\mathrm{AOQ}_{i-1}$ are independent. For $i^{\text {th }}$ stage, if a lot is accepted, $n_{i}$ sampled items are inspected and $N_{i}-n_{i}$ items are released without any inspections. However, for rejected lots a full inspection is performed and all the defectives are replaced with good ones. Therefore, AOQ of the $i^{\text {th }}$ stage is given as follows:

$$
\mathrm{AOQ}_{i}=\int_{p_{l i}}^{p_{u i}}\left(\frac{\left(N_{i}-n_{i}\right) p_{i}}{N_{i}}\right) \cdot P a_{i} \cdot f\left(p_{i}^{0} \mid a, b\right) d p_{i}^{0},
$$

where $f($.$) is the density function of the beta distribution,$ $P \alpha_{i}$ is the acceptance probability of a lot at stage $i$ and can be calculated as follows:

$$
P a_{i}=p\left(d \leq c_{i}\right)=\int_{p_{\text {li }}}^{p_{\text {ui }}} p\left(d \leq c_{i} \mid p_{i}^{0}\right) \cdot f\left(p_{i}^{0} \mid a, b\right) d p_{i}^{0}
$$

$$
=\int_{p_{l i}}^{p_{u i}} \sum_{d=0}^{c_{i}} \frac{\left(\begin{array}{c}
N \cdot p_{i} \\
d
\end{array}\right)\left(\begin{array}{c}
N-N \cdot p_{i} \\
n-d
\end{array}\right)}{\left(\begin{array}{c}
N \\
n
\end{array}\right)} f\left(p_{i}^{0} \mid a, b\right) d p_{i}^{0},
$$

$P_{l i}$ and $P_{u i}$ are the lower and upper bounds of $P^{0}{ }_{i}$ respectively. Note that $P \alpha_{i}$ is a function of $P^{0}{ }_{i}$.

Average total inspection for stage $i\left(\mathrm{ATI}_{i}\right)$ is the average number of inspected units over the accepted and rejected lots. For accepted lots only $n_{i}$ items are inspected and for rejected lots the entire lot is inspected. Hence, ATI for the stage $i$ can be calculated as follows:

$$
A T I_{i}=n_{i}+\left(1-P_{a_{i}}\right) \cdot\left(N_{i}-n_{i}\right) .
$$

Three types of costs are defined: test costs $\left(t c_{i}\right)$, rework costs $\left(r c_{i}\right)$ and the penalty cost $(p c)$. Test cost is the cost of a single inspection and according to (1) the total test cost $T T C$ can be calculated as:

$$
T T C=\sum_{i=1}^{r} r c_{i}\left(n_{i}+\left(1-P_{a_{i}}\right) \cdot\left(N_{i}-n_{i}\right)\right) .
$$

Rework or replacement cost is incurred when a defective item is discovered through testing and reworked or replaced by a good item. The average number of defective items that should be reworked or replaced at stage $i, n d_{i,}$, can be expressed as:

$$
n d_{i}=\left(n_{i} p_{i}\right) \cdot P_{a_{i}}+\left(N_{i} p_{i}\right) \cdot\left(1-P_{a_{i}}\right),
$$

Therefore, total rework cost TRC is given by:

$$
T R C=\sum_{i=1}^{r} r c_{i} \times n d_{i} .
$$

The penalty cost is incurred when a defective product is shipped to the costumer. Therefore total penalty cost TPC is given by:

$$
T P C=p c \times A O Q_{n} \times N_{n} .
$$

It is assumed that $r c_{i}<r c_{j}$, for all $i<j$. This assumption avoids having to introduce separate penalty costs for each stage. The penalty cost of detecting a defective item at stage $j$, instead of earlier at stage $i$, is derived by $r c_{j}-r c_{i}$.

The total inspection cost TIC is expressed by the sum of total test cost TTC, total rework cost TRC and total penalty cost TPC. This total inspection cost, can be applied to find the optimum inspection strategy. The objective function is shown as follows:

$$
\text { Min TIC }=\text { TTC }+ \text { TRC }+ \text { TPC }
$$

Subject to:

$$
\begin{gathered}
c_{i} \leq n_{i} \leq N_{i} \\
n_{i} ; c_{i} \text { integer } \quad i=1,2, \ldots, r .
\end{gathered}
$$

(2) shows that the sample size can vary from 0 (no inspection) to $N$ (full inspection). A sample size between 0 and $N$ means that sampling inspection is preferred. 


\section{SOLUTION APPROACH}

\subsection{Simulation Model}

Simulation can be used to study processes that are too complex to permit analytical model formulation and/or evaluation. The mathematical model, illustrated in Section 2 is too complex to analytically evaluate and find the optimum or the close to optimum inspection strategy. The complexity can be due to the stochastic nature of the defective proportion $P^{0}{ }_{i}$ and the interaction between stages' outputs. Simulation models can adequately map the real world systems, as they actually exist. In this case, simulation models are used to estimate the output performance measures of complex systems.

In this study, the popular Arena software (Kelton et al. 2007) is used to build a simulation model for the described multi-stage inspection problem. The model is verified by developing the model in a modular manner, using Arena's trace facility, substituting constants for random variables and manually checking the results.

Figure 3 presents a conceptual illustration of the proposed simulation model. This is a general framework of what exactly occurs in simulation runs for each entity, which is a simulated lot. At each stage, first the proportion of inherent defective item $P^{0}{ }_{i}$ is generated from a beta distribution with specified parameters and assigned to the lot as an attribute. Then, the actual proportion of defective items is calculated considering the outgoing quality of the prior stage. After calculating the number of defectives in the lot, the number of defectives in a sample of size $n_{i}$ is randomly generated from a hyper-geometric distribution. Since Arena does not generate random hyper-geometric numbers, a VBA code is developed inside Arena to generate the number of defectives for each sample. If this number is less than or equal to the acceptance number $c_{i}$ ,the lot is accepted and sample number and the number of defectives are used to calculate the test cost and rework cost at stage $i$. However, if the lot is rejected $\left(d>c_{i}\right)$, the cost of testing the entire lot and reworking all the defective items are calculated and assigned to the lot. In the last stage, total penalty cost is calculated using the number of outgoing defectives. Then, all of these costs are summed to calculate the total inspection cost. In each simulation replication, 50 lots are generated and the total inspection cost and other relevant outputs are averaged over the lots to estimate the expected total inspection cost and other simulation outputs such as average outgoing quality AOQ and average total inspection ATI.

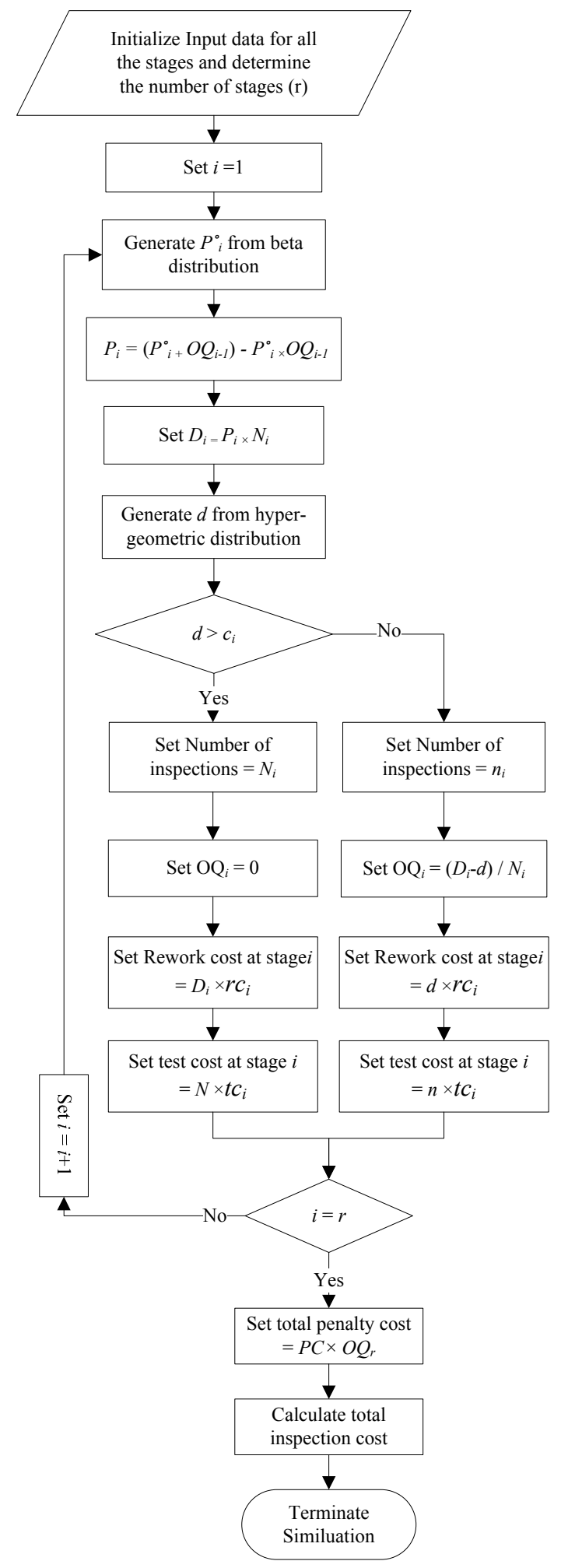

Figure 3: A conceptual illustration of the simulation model; $O Q_{i}$ is the outgoing proportion of defective items at stage $i$. 


\subsection{Simulation Optimization}

The complexities and uncertainties in real world systems are the primary reason that simulation is often chosen as a basis for handling the decision problems associated with those systems. The optimization of simulation models often deals with the situation in which the interest is to find which of a large number of sets of model specifications lead to optimal output performance (April et al. 2003). One of the popular methods to optimize simulated systems is using metaheuristics. In this mechanism, simulation model is treated as a black-box, i.e. only the inputs and outputs of the simulation model are observed. At each iteration, the metaheuristic optimizer chooses a set of values for input variables and uses the output values generated by the simulation model to make decisions regarding the selection of the next trial solution with the goal of finding optimal values for the decision variables.

In this study, we use OptQuest optimization software, which is provided with Arena, to find the optimal inspection strategy in a multi-stage process. OptQuest, combines the metaheuristics of Tabu Search, Scatter Search and Neural Networks into a single, composite search algorithm to provide maximum efficiency in identifying new scenarios (April et al. 2003, Kleijnen and Wan 2006, Glover et al. 2000). In this problem, OptQuest searches for the optimal sample size and acceptance number of each stage to minimize the expected total inspection cost as the response of the simulation model.

\section{NUMERICAL EXAMPLE}

In this section, we will use a numerical example to illustrate how our model can be applied to find the optimal inspection plan. A 5-stage serial manufacturing process, representing a stack-up assembly line is considered. Table 1 presents the parameters of the process. Penalty cost is set at 1600 and a lot size of 1000 is assumed. Beta distribution parameters are set to $a=1, b=1$ for all the stages with $p_{l}=0.006$ and $p_{u}=0.03$. An Arena model is developed based on these parameters to simulate our multistage process.

Table 1: Cost parameters

\begin{tabular}{lll}
\hline Stage & Test cost & Rework cost \\
\hline 1 & 5 & 50 \\
2 & 5 & 100 \\
3 & 15 & 200 \\
4 & 12 & 400 \\
5 & 15 & 800 \\
\hline
\end{tabular}

After building the simulation model, OptQuest is used to find the optimum input parameters, which lead to the optimum inspection strategy. Number of replications is set to vary dynamically between 5 and 10 . Figure 4 presents the coverage of the OptQuest's algorithm. The suggested values for input parameters, which determine the starting point is set to an outgoing inspection. It is shown in the figure that OptQuest has evaluated 603 different inspection strategies and the best one has discovered by the $353^{\text {th }}$ run. Notice that, the OptQuest algorithm has not been able to improve the solution for 250 runs. Therefore, the best value found in the $353^{\text {th }}$ run can be considered as the optimum or a nearly optimum solution.

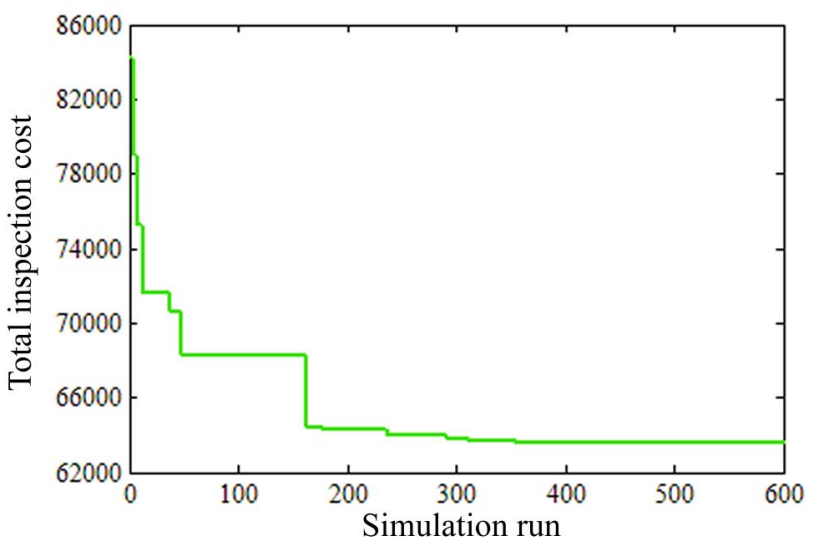

Figure 4: Final optimization window of OptQuest

Table 2 presents the optimal inspection strategy found by OptQuest. It can be seen that in stages 1 and 3 no inspection is opted. This means that the cost of avoiding the detection of defective items does not outweigh the costs of inspection. This fact can be explained by considering the relatively low rework costs compared to the test costs in these stages. In stages 2 and 4 a full inspection and in stage 5 a sampling inspection is chosen. The choice of full inspection in stages 2 and 4 implies that the cost of avoiding the detection of defectives in these stages outweighs the inspection costs. In stage 5, a sampling inspection is preferred to full inspection. This can be explained considering the high costs of reworking and testing in this stage. Therefore, sampling is opted and sampling parameters are determined so that the penalty costs and other costs are balanced.

Table 2: The optimal solution

\begin{tabular}{lll}
\hline Stage & $n$ & $c$ \\
\hline 1 & 0 & - \\
2 & 1000 & - \\
3 & 0 & - \\
4 & 1000 & - \\
5 & 151 & 2 \\
\hline
\end{tabular}

Total inspection cost and other relevant outputs of the simulation model are shown in table 3. Comparing to the 
Table 3: Outputs of the simulation model for the optimum inspection strategy

\begin{tabular}{|c|c|c|c|c|c|c|c|c|c|c|}
\hline \multirow{2}{*}{ Responses } & \multicolumn{2}{|c|}{ Stage 1} & \multicolumn{2}{|c|}{ Stage 2} & \multicolumn{2}{|c|}{ Stage 3} & \multicolumn{2}{|c|}{ Stage 4} & \multicolumn{2}{|c|}{ Stage 5} \\
\hline & Average & $\begin{array}{l}\text { Half } \\
\text { width }\end{array}$ & Average & $\begin{array}{l}\text { Half } \\
\text { width }\end{array}$ & Average & $\begin{array}{l}\text { Half } \\
\text { width }\end{array}$ & Average & $\begin{array}{l}\text { Half } \\
\text { width }\end{array}$ & Average & $\begin{array}{l}\text { Half } \\
\text { width }\end{array}$ \\
\hline AOQ & 0.0176 & .0008 & 0 & 0 & 0.01872 & 0.0003 & 0 & 0 & 0.007 & 0.0004 \\
\hline ATI & 0 & 0 & 1000 & 0 & 0 & 0 & 1000 & 0 & 575.50 & 39.253 \\
\hline Total cost & 63898 & 814.63 & & & & & & & & \\
\hline
\end{tabular}

starting point of the optimization, which is an outgoing inspection, the total inspection cost is lowered from 85020 to 63898 with an outgoing proportion of defectives in the last stage equal to 0.007 . ATI in the last stage shows that in average over accepted and rejected lots nearly half of the items of each lot are inspected. All of the outputs are provided with the half width of a $95 \%$ confidence interval on the expected value of each output. The Half Width columns are included in order to show the reliability of the results from all replications.

\section{CONCLUSIONS}

In this paper, an optimum rectifying inspection plan for multi-stage manufacturing processes was proposed. The proposed method is able to find the optimum inspection strategy, which leads to the minimum inspection cost while still assuring a required output quality. For each stage, the model is able to determine the type of inspection $(0 \%$, $100 \%$ or sampling) and also the inspection parameters for those stations which sampling should be performed in. Using a numerical example, the applicability of the proposed approach is demonstrated. It can be seen that the optimal inspection strategy contains both full and sampling inspections, therefore, the optimal inspection is not necessarily $0 \%-100 \%$ or sampling for all the stages.

Solving such problems with traditional mathematical techniques are usually time consuming and subject to rather restricting assumptions. The use of simulation modeling and optimization in the multi-stage inspection problem has allowed us to effectively solve much more complicated problems. Moreover, considering the defective ratio as a random variable has provided us with more accurate results especially where $P^{0}{ }_{i}$ is not exactly known.

The model can be extended to consider the inspection errors. We are studying the effect of errors on inspection strategies and our future work (Vaghefi and Sarhangian 2008) provides an optimum inspection strategy in the presence of misclassification errors.

\section{REFERENCES}

April, J., F. Glover, J. P. Kelly, and M. Laguna. 2003. Practical Introduction to simulation optimization. In Proceedings of the 2003 Winter Simulation Conference, ed. S. Chick, P. J. Sánchez, D. Ferrin, and D. J. Morrice, 71-78. Piscataway, New Jersey: Institute of Electrical and Electronics Engineers, Inc.

Glover, F., M. Laguna, and R. Marti. 2000. Fundamentals of scatter search and path relinking. Control and $C y$ bernetics 29(3):653-684.

Heredia-Langner, A., D. C. Montgomery, and A. W. M. Carlyle. 2002. Solving a multistage partial inspection problem using genetic algorithms. International journal of production research 40(8):1923-1940.

Kelton, W. D., R. P. Sadowski, and D. T. Sturrock. 2007. Simulation with Arena. 4rd ed. New York: McGrawHill, Inc.

Kleijnen, J. P. C., and J. Wan. 2007. Optimization of simulated systems: OptQuest and alternatives. Simulation Modelling Practice and Theory 15:354-362.

Lindsay, G. F., and A. B. Bishop. 1964. Allocation of screening inspection effort - a dynamic programming approach. Management Science 10(2):342-352.

Montgomery, D. C. 2005. Introduction to Statistical Quality Control. New York: John Wiley \& Sons, Inc.

Raz, T. 1986. A survey of models for allocating inspection effort in multistage production systems. Journal of Quality Technology 18(4):239-247.

Vaghefi, A., V. Sarhangian. 2008. An optimum inspection plan in multistage manufacturing systems with inspection errors. Working paper, Department of Industrial Engineering, Iran University of Science and Technology.

Volsem, S. V., W. Dullaert, and H. V. Landeghem. 2007. An Evolutionary Algorithm and discrete event simulation for optimizing inspection strategies for multistage processes. European Journal of Operational Research 179:621-633.

White, L. S. 1969. Shortest route models for the allocation of inspection effort on a production line. Management Science 15(5):249-259. 
Wu, Z., M. Xie, and Z. Wang. 2001. Optimum rectifying inspection plans. International journal of production research 39(8):1575-1588.

\section{AUTHOR BIOGRAPHIES}

VAHID SARHANGIAN is a researcher and student in the Department of Industrial Engineering at Iran University of Science and Technology. His research interests include Simulation Modeling and Analysis, Simulation Optimization using Metaheuristic Algorithms and Response Surface Methodology, and Multiple Criteria Decision Analysis. He is currently working on optimizing maintenance strategies for randomly failing production systems using simulation optimization. His email address is <sarhangian@ind.iust.ac.ir>.

ABOLFAZL VAGHEFI is a Ph.D. candidate in industrial engineering at Iran University of Science and Technology. His fields of interest are applied statistics including statistical methods for process monitoring and improvement and simulation modeling and analysis. He has been teaching Simulation Modeling \& Analysis in the industrial engineering and railway engineering departments at Iran University of Science and Technology since 2005. His email address is<vaghefi@iust.ac.ir>.

HAMIDREZA ESKANDARI is an Assistant Professor of Industrial Engineering at the Tarbiat Modares University, Tehran, Iran. He received his Bachelor's degree in Electrical Engineering from the University of Tehran (1998), his Master's degree in Socio-Economic Systems Engineering from the Iran University of Science and Technology (2001) and his Ph.D. in Industrial Engineering from the University of Central Florida (2006). His research interests include Simulation Modeling and Analysis, Simulation Optimization, and Evolutionary Multiobjective Optimization. His email address is <eskandari@modares.ac.ir>.

MOSTAFA K. ARDAKANI is a Postdoctoral Fellow in the Department of Civil Engineering at the Catholic University of America, Washington D.C. His research interests include applied statistics and optimization methods for robust parameter design. He is currently working on modeling human driving behavior and response with applications to intelligent agent-based traffic flow simulation, sponsored by National Science Foundation. His email address is <ardakani@cua.edu>. 